Discrete Comput Geom 36:363-379 (2006)

DOI: $10.1007 / \mathrm{s} 00454-006-1238-\mathrm{y}$

\title{
Isodiametric Problems for Polygons
}

\author{
Michael J. Mossinghoff \\ Department of Mathematics, Davidson College, \\ Davidson, NC 28035-6996, USA \\ mjm@member.ams.org
}

\begin{abstract}
The maximal area of a polygon with $n=2 m$ edges and unit diameter is not known when $m \geq 5$, nor is the maximal perimeter of a convex polygon with $n=2^{m}$ edges and unit diameter known when $m \geq 4$. We construct improved polygons in both problems, and show that the values we obtain cannot be improved for large $n$ by more than $c_{1} / n^{3}$ in the area problem and $c_{2} / n^{5}$ in the perimeter problem, for certain constants $c_{1}$ and $c_{2}$.
\end{abstract}

\section{Introduction}

The isoperimetric problem in the plane asks for the maximal area of a closed curve with fixed perimeter. If one fixes the diameter instead of the perimeter, one obtains two similar isodiametric problems: first, maximize the area, and second, for convex curves, maximize the perimeter. The circle is optimal in all three of these problems. Its optimality in the isodiametric area problem was established by Bieberbach [4], and in the perimeter problem by Rosenthal and Szász [12].

We can ask the same three questions for polygons. In the isoperimetric problem, it is again well known that the regular $n$-gon alone has maximal area among all $n$-gons with the same perimeter. We can express this as an inequality: the area $A$ of a polygon with $n$ sides and perimeter $L$ satisfies

$$
A \leq \frac{L^{2}}{4 n} \cot \left(\frac{\pi}{n}\right)
$$

The isodiametric problems for polygons however are more complicated, and both problems are described in problem B6 of [6]. In the area problem, Reinhardt [11] (see also [10]) proved that the regular $n$-gon alone is optimal when $n$ is odd. When $n=4$, the square achieves the largest possible area, but in this case there are an infinite number of quadrilaterals with the same diameter and area. Another optimal quadrilateral for example is shown in Fig. 1(a). Lenz posed the problem of determining the maximal area 


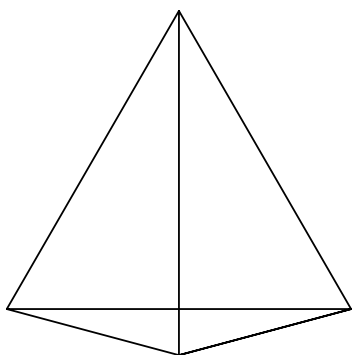

(a) $n=4$.

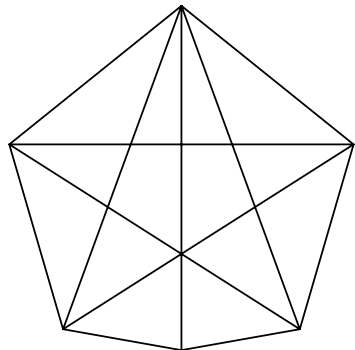

(b) $n=6$.

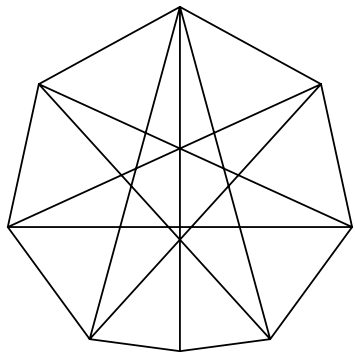

(c) $n=8$.

Fig. 1. Some optimal polygons.

when $n$ is even and $n \geq 6$ in [8]. Reinhardt in fact proved that the regular $n$-gon is never optimal for such $n$, and Schäffer [13] published another proof of this fact. Neither proof however establishes a quantitative improvement in the area problem when $n$ is even.

The optimal area is known for some small values of $n$. In 1975 Graham [7] determined the optimal hexagon, displayed in Fig. 1(b). This hexagon also appeared in 1961 in a note of Bieri [5], who showed it was optimal among hexagons with an axis of symmetry. Its area is approximately $3.9 \%$ larger than the regular hexagon with the same diameter. Graham also conjectured that the line segments of maximal length in any optimal $n$ gon with $n$ even form a similar shape: a circuit of length $n-1$, together with a single additional line segment attached to one vertex. Audet et al. [3] verified this conjecture for $n=8$ in 2002, computing the unique optimal octagon, shown in Fig. 1(c). Its area is about $2.8 \%$ larger than the regular octagon.

The isodiametric problem for the perimeter was likewise investigated by Reinhardt in [11]. He proved that the regular $n$-gon is again optimal among all convex $n$-gons if $n$ is odd. More generally, he determined the optimal perimeter in the case that $n$ has an odd factor, and it follows from his paper that a unique polygon achieves this value only if $n=p$ or $n=2 p$, for some odd prime $p$. Reinhardt also established an upper bound on the perimeter $L$ of a convex polygon with $n$ sides and diameter $d$ :

$$
L \leq 2 d n \sin \left(\frac{\pi}{2 n}\right)
$$

Reinhardt's proof is also described in [10], and another proof of this inequality appears for instance in [9]. Let $\bar{L}_{n}$ denote the value of this upper bound for the case of polygons with unit diameter,

$$
\bar{L}_{n}:=2 n \sin \left(\frac{\pi}{2 n}\right) .
$$

By combining (1) and (2), we can record an inequality relating the area of a convex polygon to its diameter and number of sides:

$$
A \leq \frac{d^{2} n}{2} \cos \left(\frac{\pi}{n}\right) \tan \left(\frac{\pi}{2 n}\right) .
$$

This is then slightly stronger than the classical isodiametric inequality for planar curves, $A \leq \pi d^{2} / 4$. Let $\bar{A}_{n}$ denote the upper bound on the area from (4) for polygons with unit 
diameter,

$$
\bar{A}_{n}:=\frac{n}{2} \cos \left(\frac{\pi}{n}\right) \tan \left(\frac{\pi}{2 n}\right) .
$$

Tamvakis [14] (see also [10]) found that the maximal perimeter of a convex quadrilateral with unit diameter is $2(1+\sqrt{2-\sqrt{3}})$, and this value is achieved only by the quadrilateral shown in Fig. 1(a). Reinhardt proved that the upper bound (2) is never achieved when $n$ is a power of 2 (this is also established in just the special case $n=8$ in [14]). It appears that little additional information on this problem was known, until very recently. In 2005 Audet et al. [1] determined the convex octagon with unit diameter and maximal perimeter. This octagon is precisely the polygon $U_{8}$ shown in Fig. 5(a) and was found independently here, though with no proof of global optimality. We remark also that Vincze posed a similar problem in [15], asking in effect for the maximal perimeter of an equilateral convex octagon with fixed diameter, and this question was recently answered by Audet et al. [2]. It is worth noting that all optimal configurations are equilateral when $n$ has an odd factor.

In Section 2 we describe a method to search for polygons with an even number of sides, fixed diameter, and large area, and with this we construct an $n$-gon with area larger than the regular polygon for each even $n \leq 20$. We then describe two general constructions that produce larger polygons for any even $n$, and these results allow us to derive a quantitative improvement in the isodiametric area problem for polygons when $n$ is even. We find in fact that there exist polygons with $n$ sides whose areas differ from the upper bound of (4) by just $O\left(1 / n^{3}\right)$, while the regular polygon has an error term of $O\left(1 / n^{2}\right)$. Let $A(P)$ denote the area of the polygon $P$, and let $P_{n}$ denote the regular polygon with $n$ sides and unit diameter. We prove the following theorem in Section 2.

Theorem 1. Let $n$ be an even integer. Let $P_{n}$ denote the regular $n$-gon with unit diameter, and let $\bar{A}_{n}$ denote the upper bound on the area of an $n$-gon with unit diameter given by (5). Then there exists a polygon $S_{n}$ with $n$ sides and unit diameter satisfying

$$
A\left(S_{n}\right)-A\left(P_{n}\right)=\frac{\pi^{3}}{16 n^{2}}+O\left(\frac{1}{n^{3}}\right)
$$

and

$$
\begin{aligned}
\bar{A}_{n}-A\left(S_{n}\right) & =\frac{(5545-456 \sqrt{114}) \pi^{3}}{5808 n^{3}}+O\left(\frac{1}{n^{4}}\right) \\
& <\frac{2 \pi^{3}}{17 n^{3}}+O\left(\frac{1}{n^{4}}\right) .
\end{aligned}
$$

In Section 3 we obtain some improvements in the isodiametric problem for the perimeter of polygons in the open cases when $n$ is a power of 2. Tamvakis [14] described a polygon $T_{n}$ whose perimeter exceeds that of the regular $n$-gon, and differs from the upper bound (2) by $O\left(1 / n^{4}\right)$. Tamvakis asked if this polygon is in fact best possible when $n=2^{m}$. We answer this question in the negative by constructing a polygon with $2^{m}$ sides whose perimeter differs from the upper bound by $O\left(1 / n^{5}\right)$. Let $L(P)$ denote the perimeter of the polygon $P$. We prove the following theorem in Section 3 . 
Theorem 2. Suppose $n=2^{m}$ with $m \geq 3$. Let $T_{n}$ denote the $n$-gon of unit diameter described in Section 3, and let $\bar{L}_{n}$ denote the upper bound on the perimeter of a convex $n$-gon with unit diameter given by (3). Then there exists a convex polygon $V_{n}$ with $n$ sides and unit diameter satisfying

$$
L\left(V_{n}\right)-L\left(T_{n}\right)=\frac{\pi^{3}}{4 n^{4}}+O\left(\frac{1}{n^{5}}\right)
$$

and

$$
\bar{L}_{n}-L\left(V_{n}\right)=\frac{\pi^{5}}{16 n^{5}}+O\left(\frac{1}{n^{6}}\right)
$$

\section{The Area Problem}

We consider the problem of constructing a polygon $P$ with $n=2 m$ sides, unit diameter, and large area. Define the skeleton of $P$ to be the set of its vertices, together with all the line segments of maximal length connecting two of its vertices. Also, define the diameter graph of $P$ to be the graph on the vertices of $P$ represented by its skeleton. We consider only polygons having the diameter graph of Graham's conjecture, and further we assume the presence of an axis of symmetry in the skeleton like that suggested by the optimal examples for $n=6$ and $n=8$.

\subsection{Decagons to Icosagons}

Suppose then that $P$ has the structure described. Let $v_{0}$ denote the lone vertex of $P$ that has degree 1 in the diameter graph, and let $v_{1}$ denote the vertex adjacent to it in the skeleton, so that $v_{0} v_{1}$ is an axis of symmetry for $P$. Label the remaining vertices so that $v_{0}, v_{1}, v_{2}, \ldots, v_{n-1}$ forms a path in the skeleton, as shown in Fig. 2.

Let $\alpha_{k}$ denote the angle formed at vertex $v_{k}$ by the segments $v_{k-1} v_{k}$ and $v_{k} v_{k+1}$. Since

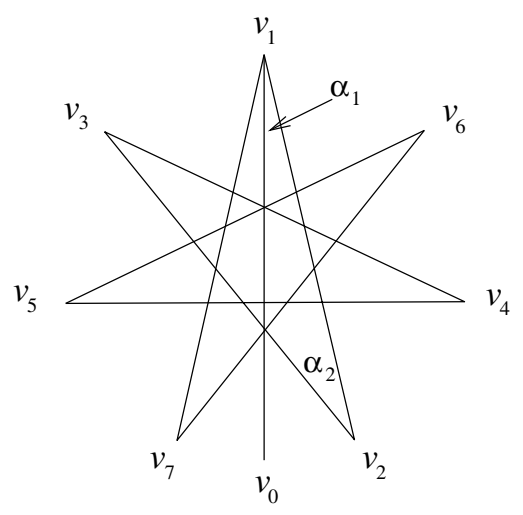

Fig. 2. Constructing a symmetric $2 m$-gon given $m-2$ angles. 
the polygon is symmetric, it may appear that we need to specify $m-1$ angles to construct the polygon. However, the position of $v_{m}$ is determined once $v_{0}, \ldots, v_{m-1}$ are placed, since $v_{m}$ must have distance 1 from $v_{m-1}$ and $v_{m} v_{m+1}$ must be a horizontal segment of length 1 . Thus, $P$ is in fact specified completely by the values of the angles $\alpha_{1}, \ldots$, $\alpha_{m-2}$.

Place $v_{0}$ at $\left(0,-\frac{1}{2}\right)$ in the plane and $v_{1}$ at $\left(0, \frac{1}{2}\right)$. Then for each $k$ we may compute the position of $\left(x_{k}, y_{k}\right)$ of vertex $v_{k}$ in terms of the angles. Let $\mathcal{R}_{\theta}$ denote the standard rotation matrix through the angle $\theta$. Since

$$
\left[\begin{array}{l}
x_{k+1}-x_{k} \\
y_{k+1}-y_{k}
\end{array}\right]=-\mathcal{R}_{\alpha_{k}}\left[\begin{array}{l}
x_{k}-x_{k-1} \\
y_{k}-y_{k-1}
\end{array}\right],
$$

it follows that

$$
\left[\begin{array}{l}
x_{k+1} \\
y_{k+1}
\end{array}\right]=\left[\begin{array}{l}
x_{k} \\
y_{k}
\end{array}\right]-(-1)^{k} \mathcal{R} \sum_{1}^{k} \alpha_{i}\left[\begin{array}{l}
0 \\
1
\end{array}\right]
$$

for $1 \leq k \leq m-2$. Thus,

$$
\begin{aligned}
& x_{k+1}=x_{k}-(-1)^{k} \sin \left(\sum_{i=1}^{k} \alpha_{i}\right), \\
& y_{k+1}=y_{k}+(-1)^{k} \cos \left(\sum_{i=1}^{k} \alpha_{i}\right) .
\end{aligned}
$$

To determine the position of $v_{m}$, note that $v_{m} v_{m+1}$ is a horizontal line segment, so

$$
\begin{aligned}
& x_{m}=\frac{(-1)^{m}}{2}, \\
& y_{m}=y_{m-1}-(-1)^{m} \sqrt{1-\left(x_{m-1}-x_{m}\right)^{2}} .
\end{aligned}
$$

We may then determine a formula for the area of $P$ as a sum of $m$ determinants whose entries are given by the points $\left(x_{k}, y_{k}\right)$. We can then construct a polygon with a rather large area by optimizing over the parameters $\alpha_{1}, \ldots, \alpha_{m-2}$. We use a heuristic optimization algorithm in Mathematica (the principal axis method, available using the F indMaximum command) to perform these calculations.

For example, when $n=6$ we have a single parameter $\alpha_{1}$, and choosing $\alpha_{1}=$ $0.3509301888 \cdots$ produces the Bieri-Graham hexagon of Fig. 1(b). When $n=8$, we construct the optimal octagon of Fig. 1(c), assuming it indeed has an axis of symmetry, by choosing $\alpha_{1}=0.2652408673 \cdots$ and $\alpha_{2}=0.4706314236 \cdots$.

We perform this analysis for even values of $n$ up to $n=20$, and obtain the polygons shown in Fig. 3. Table 1 shows the values of the angles $\left\{\alpha_{k}\right\}$ used to obtain these shapes, and Table 2 summarizes the improvements obtained in the area. For each $n$, the second column in the second table shows the area of the regular $n$-gon, $A\left(P_{n}\right)=$ $(n / 8) \sin (2 \pi / n)$. Subsequent columns display the area of the polygon $Q_{n}$ that we construct with this method, the upper bound on the area $\bar{A}_{n}$ from (5), the percentage gain in the area of $Q_{n}$ compared with that of $P_{n}$, and the fraction $g_{n}$ of the length of the interval $\left[A\left(P_{n}\right), \bar{A}_{n}\right]$ where $A\left(Q_{n}\right)$ lies, so

$$
g_{n}=\frac{A\left(Q_{n}\right)-A\left(P_{n}\right)}{\bar{A}_{n}-A\left(P_{n}\right)} .
$$



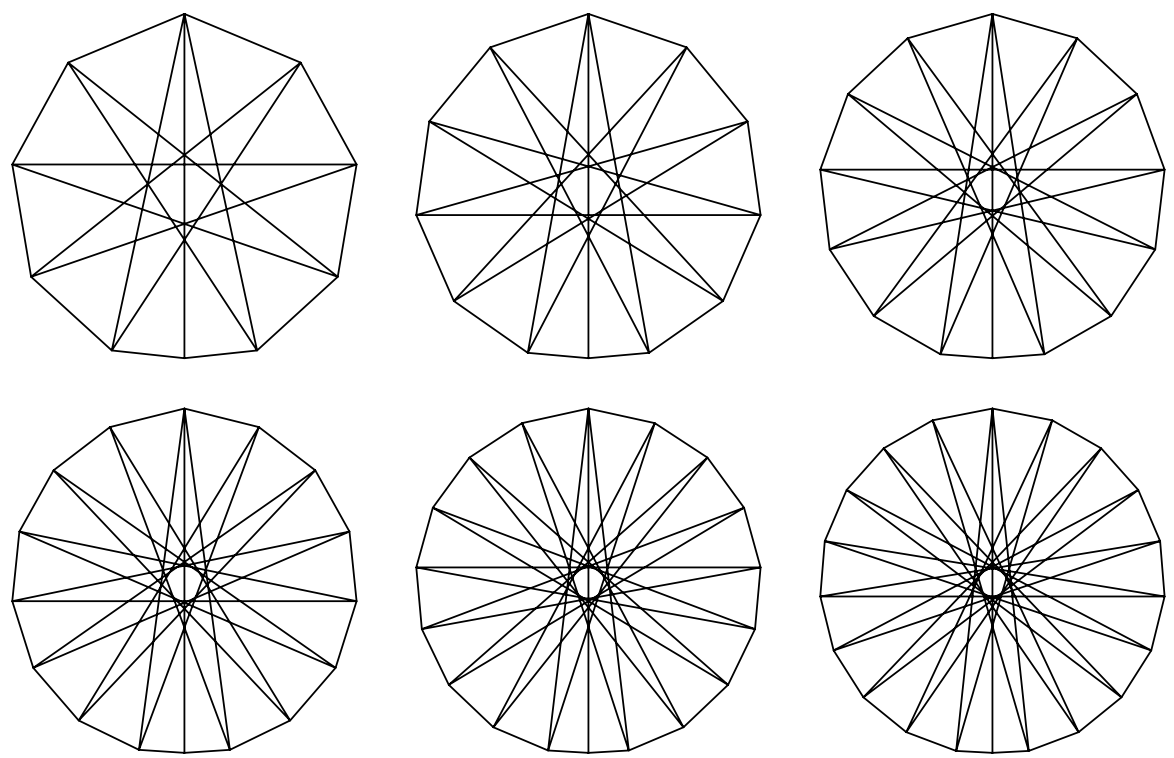

Fig. 3. Improved polygons $Q_{n}$ for $n=10$ through $n=20$.

Table 1. Angles for constructing $Q_{n}$.

\begin{tabular}{rcccccccc}
\hline$n$ & $\alpha_{1}$ & $\alpha_{2}$ & $\alpha_{3}$ & $\alpha_{4}$ & $\alpha_{5}$ & $\alpha_{6}$ & $\alpha_{7}$ & $\alpha_{8}$ \\
\hline 6 & 0.35093 & & & & & & & \\
8 & 0.26524 & 0.47063 & & & & & & \\
10 & 0.21261 & 0.36813 & 0.31861 & & & & & \\
12 & 0.17709 & 0.30260 & 0.26295 & 0.27946 & & & & \\
14 & 0.15158 & 0.25703 & 0.22390 & 0.23763 & 0.23244 & & & \\
16 & 0.13243 & 0.22345 & 0.19497 & 0.20672 & 0.20229 & 0.20401 & & \\
18 & 0.11753 & 0.19766 & 0.17265 & 0.18294 & 0.17907 & 0.18058 & 0.18000 & \\
20 & 0.10563 & 0.17723 & 0.15492 & 0.16408 & 0.16064 & 0.16198 & 0.16146 & 0.16166 \\
\hline
\end{tabular}

Table 2. Areas of the improved polygons.

\begin{tabular}{rccccc}
\hline$n$ & $A\left(P_{n}\right)$ & $A\left(Q_{n}\right)$ & $\bar{A}_{n}$ & Gain $(\%)$ & $g_{n}$ \\
\hline 6 & 0.6495190528 & 0.6749814429 & 0.6961524227 & 3.920 & 0.546 \\
8 & 0.7071067812 & 0.7268684828 & 0.7350842599 & 2.795 & 0.706 \\
10 & 0.7347315654 & 0.7491373459 & 0.7531627703 & 1.961 & 0.782 \\
12 & 0.7500000000 & 0.7607298734 & 0.7629992851 & 1.431 & 0.825 \\
14 & 0.7592965435 & 0.7675310111 & 0.7689359584 & 1.084 & 0.854 \\
16 & 0.7653668647 & 0.7718613220 & 0.7727913493 & 0.849 & 0.875 \\
18 & 0.7695453225 & 0.7747881651 & 0.7754356273 & 0.681 & 0.890 \\
20 & 0.7725424859 & 0.7768587560 & 0.7773275822 & 0.559 & 0.902 \\
\hline
\end{tabular}


It is not surprising that the percentage area gained in the polygons we construct diminishes with $n$. After all,

$$
\bar{A}_{n}=\frac{\pi}{4}-\frac{5 \pi^{3}}{48 n^{2}}+\frac{\pi^{5}}{480 n^{4}}+O\left(\frac{1}{n^{6}}\right),
$$

and, for $n$ even,

$$
A\left(P_{n}\right)=\frac{\pi}{4}-\frac{\pi^{3}}{6 n^{2}}+\frac{\pi^{5}}{30 n^{4}}+O\left(\frac{1}{n^{6}}\right),
$$

so $\bar{A}_{n}-A\left(P_{n}\right) \sim \pi^{3} / 16 n^{2}$. However, it is interesting to note that the last column of Table 2 indicates that $A\left(Q_{n}\right)$ appears to approach $\bar{A}_{n}$ much faster than $A\left(P_{n}\right)$ does as $n$ increases. In fact, a least-squares fit on the data for $10 \leq n \leq 20$ suggests that the quantity $\bar{A}_{n}-A\left(Q_{n}\right)$ behaves like $5.05 / n^{3.10}$. We can verify this trend, and establish a quantitative improvement in the isodiametric problem for the area when $n$ is even.

\subsection{General Construction}

The method just described requires optimizing an expression in $(n / 2)-2$ variables to determine a favorable area for a polygon with $n$ sides, so clearly we must modify this technique for the general case.

Studying the examples in Fig. 3 and the data in Table 1, we see that most of the angles $\alpha_{2}, \alpha_{3}, \ldots, \alpha_{m-2}$ have rather similar values. In fact, these values appear to exhibit a pattern of damped oscillation, converging in an alternating manner to some mean value. For large $n$, then, we expect most of these angles to be very nearly equal, so as a first approximation, we assume that in fact $\alpha_{2}=\alpha_{3}=\cdots=\alpha_{m-2}$. Denote this value by $\beta$, and for simplicity we use $\alpha$ to denote the value of the angle $\alpha_{1}$. We may then ask for the optimal values of $\alpha$ and $\beta$, for fixed $n$, that maximize the area of an $n$-gon constructed in this way.

To compute this, we derive a formula for the area of the polygon in terms of $\alpha$ and $\beta$. Fix an even integer $n=2 m$. Again, write $\left(x_{k}, y_{k}\right)$ for the location of vertex $v_{k}$, and place $v_{0}$ at $(0, h-1)$ and $v_{1}$ at $(0, h)$, where $h$ is a parameter to be selected later. We find from (6) that

$$
\begin{aligned}
x_{k} & =\sum_{j=0}^{k-2}(-1)^{j} \sin (\alpha+j \beta) \\
& =\operatorname{Im}\left(\sum_{j=0}^{k-2}(-1)^{j} e^{i(\alpha+j \beta)}\right) \\
& =\frac{\operatorname{Im}\left(e^{i \alpha}\left(1+(-1)^{k} e^{i(k-1) \beta}\right)\left(1+e^{-i \beta}\right)\right)}{2(1+\cos \beta)}
\end{aligned}
$$

for $1 \leq k<m$. Define $r_{k}$ and $s_{k}$ by

$$
2 r_{k}=1+(-1)^{k} \cos ((k-1) \beta)+(-1)^{k} \frac{\sin \beta}{1+\cos \beta} \sin ((k-1) \beta)
$$


and

$$
2 s_{k}=(-1)^{k} \sin ((k-1) \beta)-\frac{\sin \beta}{1+\cos \beta}\left(1+(-1)^{k} \cos ((k-1) \beta)\right) .
$$

We find that

$$
x_{k}=r_{k} \sin \alpha+s_{k} \cos \alpha,
$$

and a similar computation reveals that

$$
y_{k}=h-r_{k} \cos \alpha+s_{k} \sin \alpha
$$

for $1 \leq k<m$. The values of $x_{m}$ and $y_{m}$ are given by (7), and we now select $h$ so that $y_{m}=0$, so

$$
h=r_{m-1} \cos \alpha-s_{m-1} \sin \alpha+(-1)^{m} \sqrt{1-\left(x_{m-1}-(-1)^{m} / 2\right)^{2}} .
$$

Let $A_{k}$ denote the area of the parallelogram with corners at the origin, the vertices $v_{k-1}$ and $v_{k+1}$, and the point $\left(x_{k-1}+x_{k+1}, y_{k-1}+y_{k+1}\right)$, so that the area of the polygon determined by $\alpha$ and $\beta$ is $\sum_{k=1}^{m} A_{k}$. Now

$$
A_{1}=\operatorname{det}\left[\begin{array}{ll}
x_{0} & x_{2} \\
y_{0} & y_{2}
\end{array}\right]=\operatorname{det}\left[\begin{array}{cc}
0 & \sin \alpha \\
h-1 & h-\cos \alpha
\end{array}\right]=(1-h) \sin \alpha .
$$

Next, by the construction and the fact that $y_{m}=0$, we see that $x_{k}$ and $y_{k}$ have opposite signs for $2 \leq k<m$, and further that $\left|y_{k+1}\right|<\left|y_{k-1}\right|$ and $\left|x_{k+1}\right|>\left|x_{k-1}\right|$ for $2 \leq k<m$, so $x_{k-1} y_{k+1}>x_{k+1} y_{k-1}$ in this range. Thus, for $2 \leq k \leq m-2$, we find that

$$
\begin{aligned}
A_{k} & =\operatorname{det}\left[\begin{array}{ll}
x_{k-1} & x_{k+1} \\
y_{k-1} & y_{k+1}
\end{array}\right] \\
& =\left(r_{k-1}-r_{k+1}\right) h \sin \alpha+\left(s_{k-1}-s_{k+1}\right) h \cos \alpha+r_{k-1} s_{k+1}-r_{k+1} s_{k-1} .
\end{aligned}
$$

The value of $r_{k-1} s_{k+1}-r_{k+1} s_{k-1}$ is

$$
\frac{\sin (2 \beta)+(-1)^{k-1}(\sin (k \beta)-\sin ((k-2) \beta))}{2(1+\cos \beta)},
$$

SO

$$
\begin{aligned}
\sum_{k=2}^{m-2} A_{k}= & \left(r_{1}+r_{2}-r_{m-2}-r_{m-1}\right) h \sin \alpha+\left(s_{1}+s_{2}-s_{m-2}-s_{m-1}\right) h \cos \alpha \\
& +\frac{1}{2(1+\cos \beta)}\left((m-3) \sin (2 \beta)-\sum_{k=2}^{m-2}(-1)^{k} \sin (k \beta)+\sum_{k=0}^{m-4}(-1)^{k} \sin (k \beta)\right) \\
= & \left(1-r_{m-2}-r_{m-1}\right) h \sin \alpha-\left(s_{m-2}+s_{m-1}\right) h \cos \alpha+\frac{(m-3) \sin (2 \beta)}{2(1+\cos \beta)} \\
& -\frac{1}{2(1+\cos \beta)}\left(\sin \beta+(-1)^{m}(\sin ((m-2) \beta)-\sin ((m-3) \beta))\right) .
\end{aligned}
$$




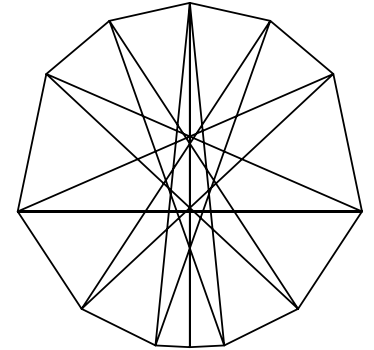

(a) $(0.1,0.24)$

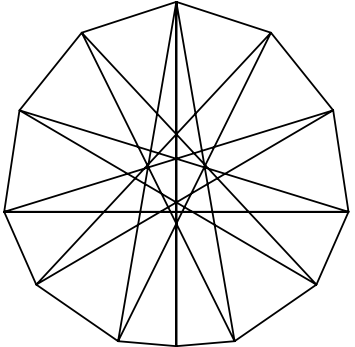

(b) $(0.17,0.29)$

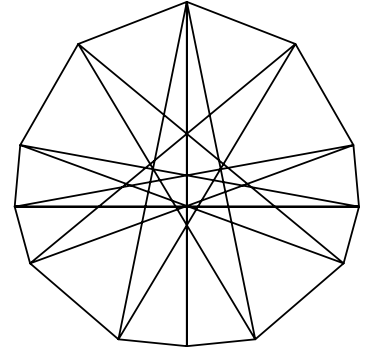

(c) $(0.2,0.34)$

Fig. 4. Dodecagons constructed for certain $(\alpha, \beta)$.

Finally, since $\left(x_{m}, y_{m}\right)=\left((-1)^{m} / 2,0\right)$ and $\left(x_{m+1}, y_{m+1}\right)=\left((-1)^{m-1} / 2,0\right)$, we have

$$
A_{m-1}+A_{m}=(-1)^{m}\left(y_{m-1}-y_{m-2}\right) / 2 .
$$

Combining these expressions, we obtain a closed formula for the area in terms of $\alpha$ and $\beta$.

Figure 4 shows three dodecagons obtained using different values for $\alpha$ and $\beta$ when $n=12$. The areas of these polygons are approximately $0.75354,0.75981$, and 0.75478 , respectively.

We may now search for values of $\alpha$ and $\beta$ (depending on $n$ ) that produce especially good values for the area. Since the sum of the angles at the points of a star is $\pi$, we certainly require $\beta=\pi / n+O\left(1 / n^{2}\right)$. Consider then setting $\alpha=s \pi / n+t \pi / n^{2}$ and $\beta=\pi / n+u \pi / n^{2}$, where $s, t$, and $u$ are free parameters. Using Mathematica to compute the asymptotic expansion in $n$ for the resulting area, we find that the optimal expression occurs when $s=t=\frac{1}{2}$ and $u=1$, which produces

$$
\sum_{k=1}^{m} A_{k}=\frac{\pi}{4}-\frac{5 \pi^{3}}{48 n^{2}}-\frac{7 \pi^{3}}{48 n^{3}}-\frac{\pi^{3}\left(60-\pi^{2}\right)}{480 n^{4}}+O\left(\frac{1}{n^{5}}\right) .
$$

Comparing this with (8) and (9), we see that the value here is substantially better than the area of the regular $n$-gon, and substantially closer to the upper bound $\bar{A}_{n}$.

The first four terms of the expression (12) are unaffected when terms of higher order are added to the values for $\alpha$ and $\beta$, so setting

$$
\beta=\frac{\pi}{n} \sum_{k \geq 0} n^{-k}=\frac{\pi}{n-1}
$$

and $\alpha=\pi /(2 n-2)$ produces the same asymptotic expression. However, in this case the star formed by the circuit of diagonals of maximal length in the polygon agrees precisely with the skeleton of the regular polygon with $n-1$ sides. The polygon we obtain thus has a very simple description: it is produced by adding a single vertex at distance 1 antipodal to an existing vertex in the regular polygon $P_{n-1}$. We denote this polygon by $R_{n}$; note that Fig. 1(a) shows the polygon $R_{4}$. We find that

$$
A\left(R_{n}\right)=\frac{1}{2} \tan \left(\frac{\pi}{2 n-2}\right)\left((n-2) \cos \left(\frac{\pi}{n-1}\right)+2 \cos \left(\frac{\pi}{2 n-2}\right)-1\right) .
$$


Observing that

$$
\bar{A}_{n}-A\left(R_{n}\right) \sim \frac{7 \pi^{3}}{48 n^{3}}
$$

confirms our observations from the data in Table 2 .

It is natural then to ask if the polygon $R_{n}$ might itself be optimal for large $n$, but in fact this is not the case. By perturbing the values of the $1 / n^{3}$ terms in the expressions for $\alpha$ and $\beta$, we may improve the coefficient of the $1 / n^{5}$ term in the expression for the area by $9 \pi^{3}\left(13 \pi^{2}+12 \pi+20\right) / 1792$. However, we can improve our construction more significantly by amending our analysis, introducing another free variable to allow for some oscillation in the angles like the pattern we observed in Table 1.

\subsection{Proof of Theorem 1}

Table 1 shows that most of the variation in the angles $\alpha_{k}$ occurs for small $k$, so as a second approximation to these numbers, we set $\alpha_{2}=\beta+\gamma$ and $\alpha_{3}=\beta-\gamma$, where $\gamma$ is a third free parameter, and maintain $\alpha_{k}=\beta$ for $4 \leq k \leq m-2$. Following our earlier strategy, we may then derive a formula for the area of the polygon in terms of $\alpha, \beta$, and $\gamma$. Let $\left(x_{k}^{\prime}, y_{k}^{\prime}\right)$ denote the coordinates of the vertex $v_{k}^{\prime}$ of the polygon. Using (10) and (11), we find that $\left(x_{k}^{\prime}, y_{k}^{\prime}\right)=\left(x_{k}, y_{k}\right)$ for $0 \leq k \leq 2$, then

$$
\begin{aligned}
& x_{k}^{\prime}=x_{k}+\sin (\alpha+\beta)-\sin (\alpha+\beta+\gamma), \\
& y_{k}^{\prime}=y_{k}-\cos (\alpha+\beta)+\cos (\alpha+\beta+\gamma)
\end{aligned}
$$

for $3 \leq k<m$, and $\left(x_{m}^{\prime}, y_{m}^{\prime}\right)$ is calculated in the same manner as $\left(x_{m}, y_{m}\right)$. The areas $A_{k}^{\prime}$ of the associated parallelograms are

$$
\begin{aligned}
& A_{1}^{\prime}=A_{1}, \\
& A_{2}^{\prime}=A_{2}-h(\sin (\alpha+\beta)-\sin (\alpha+\beta+\gamma)), \\
& A_{3}^{\prime}=A_{3}-x_{2}(\cos (\alpha+\beta)-\cos (\alpha+\beta+\gamma))-y_{2}(\sin (\alpha+\beta)-\sin (\alpha+\beta+\gamma)),
\end{aligned}
$$

and

$$
\begin{aligned}
A_{k}^{\prime}= & A_{k}+(\sin (\alpha+\beta)-\sin (\alpha+\beta+\gamma))\left(y_{k+1}-y_{k-1}\right) \\
& +(\cos (\alpha+\beta)-\cos (\alpha+\beta+\gamma))\left(x_{k+1}-x_{k-1}\right)
\end{aligned}
$$

for $4 \leq k \leq m-2$, so

$$
\begin{aligned}
\sum_{k=4}^{m-2} A_{k}^{\prime}= & \sum_{k=4}^{m-2} A_{k}+(\sin (\alpha+\beta)-\sin (\alpha+\beta+\gamma))\left(y_{m-1}+y_{m-2}-y_{4}-y_{3}\right) \\
& +(\cos (\alpha+\beta)-\cos (\alpha+\beta+\gamma))\left(x_{m-1}+x_{m-2}-x_{4}-x_{3}\right) .
\end{aligned}
$$

Finally, choosing $h$ again so that $y_{m}^{\prime}=0$, we have

$$
A_{m-1}^{\prime}+A_{m}^{\prime}=\frac{(-1)^{m}\left(y_{m-1}^{\prime}-y_{m-2}^{\prime}\right)}{2}=A_{m-1}+A_{m} .
$$


We set

$$
\begin{aligned}
& \alpha=\frac{s \pi}{n}+\frac{t \pi}{n^{2}}, \\
& \beta=\frac{\pi}{n}+\frac{u \pi}{n^{2}}, \quad \text { and } \\
& \gamma=\frac{v \pi}{n}+\frac{w \pi}{n^{2}},
\end{aligned}
$$

with $s, t, u, v$, and $w$ free parameters. With these parameters, the asymptotic expansion for the area as $n$ grows large is

$$
\frac{\pi}{4}-\left(\frac{\pi(2 s-1-4 v)}{2 n}\right)^{3 / 2}+O\left(\frac{1}{n^{2}}\right)
$$

and we therefore set $v=(2 s-1) / 4$ to obtain a series at least as good as the one for the area of $R_{n}$. Heuristic optimizations in the remaining parameters, combined with observations on the terms in the asymptotic expansion for the area, then suggest the additional relations $u=2(1-s)$ and $w=(s+t-1) / 2$. With these simplifications, the expression for the area is

$$
\frac{\pi}{4}-\frac{5 \pi^{3}}{48 n^{2}}-\frac{\pi^{3}}{192 n^{3}}\left(88 s^{3}+84 s^{2}-222 s+107\right)+O\left(\frac{1}{n^{4}}\right),
$$

and the $1 / n^{3}$ term is maximized for $s>0$ when $s=(2 \sqrt{114}-7) / 22=0.65246165 \cdots$, and thus $u=0.69507668 \cdots$ and $v=0.076230829 \cdots$. After this, the final free parameter, $t$, first affects the $1 / n^{5}$ term of the series, but its value is still significant for small $n$. This term is maximized by selecting

$$
t=\frac{44(103104 \sqrt{114}-998743)+(-1)^{n / 2} 75 \pi(347 \sqrt{114}-714)}{8811220},
$$

so $t=0.58986270 \cdots$ and $w=0.12116218 \cdots$ when $n \equiv 0 \bmod 4$, and $t=$ $0.42990185 \cdots$ and $w=0.041181759 \cdots$ when $n \equiv 2 \bmod 4$. We then obtain a polygon $S_{n}$ whose area satisfies

$$
A\left(S_{n}\right)=\frac{\pi}{4}-\frac{5 \pi^{3}}{48 n^{2}}-\frac{(5545-456 \sqrt{114}) \pi^{3}}{5808 n^{3}}+O\left(\frac{1}{n^{4}}\right) .
$$

Since

$$
\frac{5545-456 \sqrt{114}}{5808}=0.11643462 \cdots<\frac{2}{17},
$$

the area of $S_{n}$ thus shows a significant improvement over the value (12) for $R_{n}$, relative to the maximum value $\bar{A}_{n}$.

Last, we verify that $S_{n}$ indeed has unit diameter. We calculate that the distance between vertices $v_{j}$ and $v_{k}$ is

$$
\left\|v_{j}-v_{k}\right\|= \begin{cases}\cos ^{2}\left(\frac{(j-k) \beta}{2}\right) \sec ^{2}\left(\frac{\beta}{2}\right), & \text { if } \quad j \neq k \bmod 2, \\ \sin ^{2}\left(\frac{(j-k) \beta}{2}\right) \sec ^{2}\left(\frac{\beta}{2}\right), & \text { if } \quad j \equiv k \bmod 2\end{cases}
$$


Table 3. Areas of the improved polygons.

\begin{tabular}{rccc}
\hline$n$ & $A\left(R_{n}\right)$ & $A\left(S_{n}\right)$ & $A\left(Q_{n}\right)$ \\
\hline 6 & 0.6722882584 & 0.6741124756 & 0.6749814429 \\
8 & 0.7253199909 & 0.7262806311 & 0.7268684828 \\
10 & 0.7482573378 & 0.7490291363 & 0.7491373459 \\
12 & 0.7601970055 & 0.7606471438 & 0.7607298734 \\
14 & 0.7671877750 & 0.7675035228 & 0.7675310111 \\
16 & 0.7716285345 & 0.7718386481 & 0.7718613220 \\
18 & 0.7746235089 & 0.7747776809 & 0.7747881651 \\
20 & 0.7767382147 & 0.7768497848 & 0.7768587560 \\
\hline
\end{tabular}

for integers $j$ and $k$ with $3 \leq j, k<m$, where $\beta$ is given in (13), and this distance is less than 1 when $|j-k|>1$. Additional calculations verify that the distance from a vertex $v$ to one of the vertices $v_{0}, v_{1}, v_{2}$, or $v_{m}$ is less than $1-c / n^{2}$ for a positive constant $c$, provided of course that $v$ is not adjacent to the vertex in question in the diameter graph. Other pairs of vertices may be checked by using symmetry and geometric arguments. This establishes Theorem 1.

Table 3 shows the area of $S_{n}$, along with the areas of the polygons $Q_{n}$ from Fig. 3 and $R_{n}$ from Section 2.2, for $6 \leq n \leq 20$.

Table 4 displays the coordinates of $(n / 2)-1$ vertices of each polygon $Q_{n}$, placing the vertices that lie on the line of symmetry at $(0,0)$ and $(0,1)$. The table lists only the vertices having positive $x$-coordinate, and shows ten digits of accuracy. We include

Table 4. Vertices $(x, y)$ of $Q_{n}$ with $x>0$.

\begin{tabular}{clll}
\hline $4\left(\frac{1}{2}, 1-\sqrt{3} / 2\right)$ & & \\
$6\left(\frac{1}{2}, 0.5976493037\right)$ & $(0.3437714530,0.06094665323)$ & \\
$8(0.4090922743,0.7762161353)$ & $\left(\frac{1}{2}, 0.3596213239\right)$ & $(0.2621417200,0.03497061254)$ \\
$10(0.3376320746,0.8585725386)$ & $\left(\frac{1}{2}, 00.5626799009\right)$ & $(0.4452924921,0.2364558913)$ \\
$(0.2110120385,0.02251653743)$ & & \\
$12(0.2853427808,0.9027767489)$ & $(0.4623140789,0.6876660959)$ & $\left(\frac{1}{2}, 0.4157256362\right)$ \\
$(0.3908898116,0.1660885646)$ & $(0.1761613676,0.01563869815)$ & \\
$14(0.2463303123,0.9291410859)$ & $(0.4195768715,0.7673152402)$ & $\left(\frac{1}{2}, 0.5476706379\right)$ \\
$(0.4728001271,0.3160245442)$ & $(0.3448430847,0.1225965957)$ & $(0.1510029070,0.01146668136)$ \\
16 & $(0.2163699626,0.9460975354)$ & $(0.3801143684,0.8205295739)$ & $(0.4793490590,0.6426819513)$ \\
$\left(\frac{1}{2}, 0.4405052446\right)$ & $(0.4389874374,0.2468813961)$ & $(0.3070352165,0.09401360842)$ \\
$(0.1320413758,0.008755794432)$ & & \\
18 & $(0.1927387186,0.9576351273)$ & $(0.3456144726,0.8576246712)$ & $(0.4519510495,0.7119805008)$ \\
$\left(\frac{1}{2}, 0.5385383592\right)$ & $(0.4838177682,0.3593663778)$ & $(0.4055713556,0.1975338808)$ \\
$(0.2759883164,0.07429206451)$ & $(0.1172628713,0.006899089214)$ & \\
20 & $(0.1736677084,0.9658354978)$ & $(0.3159107016,0.8844267027)$ & $(0.4234853329,0.7635680874)$ \\
$(0.4869692602,0.6150850237)$ & $\left(\frac{1}{2}, 0.4541761074\right)$ & $(0.4612553307,0.2974844489)$ \\
$(0.3748185315,0.1613132078)$ & $(0.2502635809,0.06014114427)$ & $(0.1054326202,0.005573550940)$ \\
\hline
\end{tabular}


the coordinates for the polygons from Fig. 1 as well for completeness, and also because some of the coordinates for $n=8$ differ slightly from those reported in [3], which has for example $(0.40980,0.77558)$ where we obtain $(0.40909,0.77622)$. However, the optimal area for an octagon computed here agrees with the value in [3], up to the precision stated in that article.

\section{The Perimeter Problem}

Recall that a Reuleaux polygon is a closed curve of constant width in the plane whose boundary consists of a finite number of circular arcs. In particular, a Reuleaux triangle with unit width can be constructed by replacing each edge of an equilateral triangle having unit edge length with a convex circular arc of radius 1 . Let $T_{n}$ denote the convex polygon with $n$ sides obtained by subdividing each bounding arc of such a Reuleaux triangle into either $\lfloor n / 3\rfloor$ or $\lceil n / 3\rceil$ subarcs of equal length, then taking the convex hull of the endpoints of these arcs. For example, Fig. 1(a) illustrates $T_{4}$. If $3 \mid n$, then the polygon $T_{n}$ achieves the upper bound in the isodiametric problem for the perimeter. Tamvakis asked if this polygon is also optimal in the open case when $n$ is a power of 2 . Its perimeter is

$$
\begin{aligned}
& L\left(T_{n}\right)= \begin{cases}\frac{4}{3}(n-1) \sin \left(\frac{\pi}{2 n-2}\right)+\frac{2}{3}(n+2) \sin \left(\frac{\pi}{2 n+4}\right) & \text { if } \quad n \equiv 1 \bmod 3 \\
\frac{2}{3}(n-2) \sin \left(\frac{\pi}{2 n-4}\right)+\frac{4}{3}(n+1) \sin \left(\frac{\pi}{2 n+2}\right) & \text { if } \quad n \equiv 2 \bmod 3\end{cases} \\
& =\pi-\frac{\pi^{3}}{24 n^{2}}+\left(\frac{\pi^{5}}{1920}-\frac{\pi^{3}}{4}\right) \frac{1}{n^{4}}+O\left(\frac{1}{n^{5}}\right) .
\end{aligned}
$$

From (3), we have that the upper bound on the perimeter of a convex polygon of unit diameter satisfies

$$
\bar{L}_{n}=\pi-\frac{\pi^{3}}{24 n^{2}}+\frac{\pi^{5}}{1920 n^{4}}+O\left(\frac{1}{n^{6}}\right) .
$$

Thus,

$$
\bar{L}_{n}-L\left(T_{n}\right) \sim \frac{\pi^{3}}{4 n^{4}} .
$$

We next describe the construction of better polygons in the isodiametric problem for the perimeter in three specific cases, then using this information we analyze the general case and establish Theorem 2.

\subsection{Octagons, Hexadecagons, and Triacontakaidigons}

We describe the construction of some improved examples in the perimeter problem for $n=8, n=16$, and $n=32$. When $n=8$, we find that $Q_{8}$ in Fig. 1(c) already has a larger 


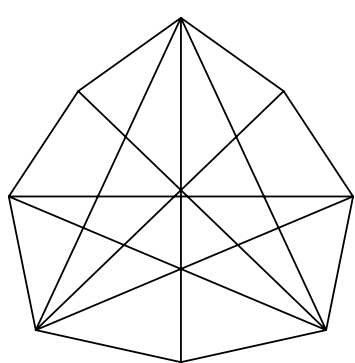

(a) $U_{8}$

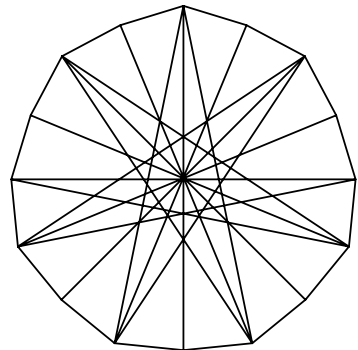

(b) $U_{16}$

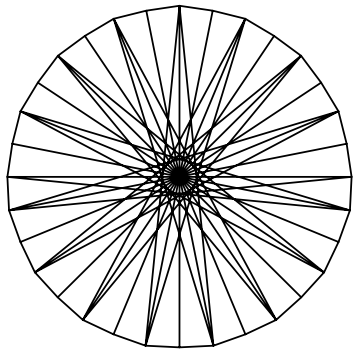

(c) $U_{32}$

Fig. 5. Improved perimeters.

perimeter than $T_{8}$, as $L\left(Q_{8}\right)=3.11924 \cdots$ and $L\left(T_{8}\right)=3.11905 \cdots$. We can improve this further by adjusting the angles $\alpha_{1}$ and $\alpha_{2}$ from our parameterization of Section 2.1 to maximize the perimeter rather than the area, creating a polygon $Q_{8}^{\prime}$ with still larger perimeter, $L\left(Q_{8}^{\prime}\right)=3.11959 \cdots$. However, we can improve this value still further by considering polygons with a very different diameter graph.

Suppose then that the line segments of maximal length in the polygon form a circuit of length $(n / 2)+1$, plus $(n / 2)-1$ additional pendant edges, arranged so that all but two particular vertices of the circuit have a pendant edge attached. For example, Fig. 1(a) exhibits this diameter graph when $n=4$, and Fig. 5 illustrates it in the cases $n=8$, $n=16$, and $n=32$.

We assume again the existence of an axis of symmetry, and we also assume that each pendant edge in the skeleton incident to a vertex bisects the angle formed by the circuit at that vertex. Suppose $n=8 m$. Then describing a polygon of unit diameter having a skeleton of this shape requires specifying $2 m-1$ angles. The coordinates of the vertices of the star shape may then be computed in terms of the parameters $\alpha_{1}, \ldots$, $\alpha_{2 m-1}$ using (6) and (7) as in Section 2.1. The coordinates of most of the pendant edges can be calculated in a similar way by modifying (6) slightly, replacing one angle $\alpha_{k}$ with $\alpha_{k} / 2$. Determining the last pair of pendant edges requires first calculating the final angle $\alpha_{2 m}$ of the star, but this is easily done.

We may then use the heuristic optimization methods in Mathematica to determine values for the angles that maximize the perimeter of this family of polygons. Our calculations produce the polygons $U_{n}$ for $n=8, n=16$, and $n=32$, and these are displayed in Fig. 5. The angles used to construct each of these polygons appear in Table 5, and the coordinates of $(n / 2)-1$ vertices of each $U_{n}$ are shown in Table 6 . In this table we again place the polygon in the plane so that the line of symmetry lies on the $y$-axis, with one

Table 5. Angles for constructing $U_{n}$.

\begin{tabular}{rccccccc}
\hline$n$ & \multicolumn{1}{c}{$\alpha_{1}$} & $\alpha_{2}$ & $\alpha_{3}$ & $\alpha_{4}$ & $\alpha_{5}$ & $\alpha_{6}$ & $\alpha_{7}$ \\
\hline 8 & 0.43528 & & & & & & \\
16 & 0.20123 & 0.38396 & 0.39975 & & & & \\
32 & 0.098779 & 0.19517 & 0.19747 & 0.19535 & 0.19721 & 0.19569 & 0.19682 \\
\hline
\end{tabular}


Table 6. Vertices $(x, y)$ of $U_{n}$ with $x>0$.

\begin{tabular}{rlll}
\hline 8 & $(0.2983440841,0.7872130729)$ & $\left(\frac{1}{2}, 0.4812353606\right)$ & $(0.4216647815,0.09324820816)$ \\
16 & $(0.1832794476,0.9438638727)$ & $(0.3524809686,0.8537890926)$ & $(0.4437023491,0.6821605336)$ \\
& $\left(\frac{1}{2}, 0.4961275338\right)$ & $(0.4807511593,0.3008657515)$ & $(0.3543830964,0.1464396785)$ \\
& $(0.1998703223,0.02017764147)$ & & \\
32 & $(0.09648737439,0.9856569151)$ & $(0.1911182156,0.9619811598)$ & $(0.2748506417,0.9117602541)$ \\
& $(0.3532863039,0.8536129803)$ & $(0.4115300344,0.7750405408)$ & $(0.4618195463,0.6911541570)$ \\
$(0.4856218616,0.5960644423)$ & $\left(\frac{1}{2}, 0.4991011855\right)$ & $(0.4951841855,0.4010785508)$ \\
$(0.4620535223,0.3084551197)$ & $(0.4199827788,0.2195349680)$ & $(0.3538052126,0.1464909348)$ \\
$(0.2807583222,0.08031652227)$ & $(0.1915518678,0.03809609805)$ & $(0.09861807059,0.004874642996)$
\end{tabular}

vertex at $(0,0)$ and another at $(0,1)$. Only the vertices with positive $x$-coordinate are listed in the table.

Table 7 displays the perimeter of each $U_{n}$, along with the perimeters of some other polygons, and the upper bound $\bar{L}_{n}$.

\subsection{Proof of Theorem 2}

The data in Table 5 suggest that the optimal values for the angles $\alpha_{2}, \ldots, \alpha_{2 m-1}$ in the polygon $U_{n}$ again exhibit a pattern of damped oscillation, with mean value approximately $2 \pi / n$. So, just as in the area problem, as an approximation we set $\alpha_{2}=\cdots=\alpha_{2 m-1}$. Denote this value by $\beta$, and write $\alpha$ for $\alpha_{1}$. Then we can determine an expression for the perimeter in terms of $\alpha$ and $\beta$. For convenience, we consider the edges on just one side of the axis of symmetry. Let $n=8 m$.

The edge directly opposite the angle $\alpha$ has length $2 \sin (\alpha / 2)$, and $4 m-4$ edges lie opposite an angle of size $\beta / 2$, each one with length $2 \sin (\beta / 4)$. This leaves just three edges to compute. In Fig. 5 these edges are the two just above the horizontal line segment on one side of the axis, and the single edge just below it. Referring to Fig. 6, we see that the coordinates of $v_{2 m-1}=\left(x_{2 m-1}, y_{2 m-1}\right)$ and $v_{2 m}=\left(x_{2 m}, y_{2 m}\right)$ are given by (10) and (11) (taking $\left.h=\frac{1}{2}\right)$, and that $v_{2 m+1}=\left(-\frac{1}{2}, y_{2 m+1}\right)$, where

$$
y_{2 m+1}=y_{2 m}+\sqrt{1-\left(x_{2 m}+\frac{1}{2}\right)^{2}},
$$

and $v_{2 m+2}=\left(\frac{1}{2}, y_{2 m+1}\right)$. Also, we may obtain $w$ by rotating $v_{2 m-1}$ about $v_{2 m}$ by $\alpha_{m} / 2$, so

$$
w-v_{2 m}=\mathcal{R}_{\theta}\left(v_{2 m-1}-v_{2 m}\right)
$$

Table 7. Perimeters of the improved polygons.

\begin{tabular}{rccccc}
\hline$n$ & $L\left(P_{n}\right)$ & $L\left(T_{n}\right)$ & $L\left(V_{n}\right)$ & $L\left(U_{n}\right)$ & $\bar{L}_{n}$ \\
\hline 8 & 3.061467459 & 3.119054312 & 3.120975785 & 3.121147134 & 3.121445152 \\
16 & 3.121445152 & 3.136438178 & 3.136532024 & 3.136543956 & 3.136548491 \\
32 & 3.136548491 & 3.140323421 & 3.140330614 & 3.140331086 & 3.140331157 \\
\hline
\end{tabular}




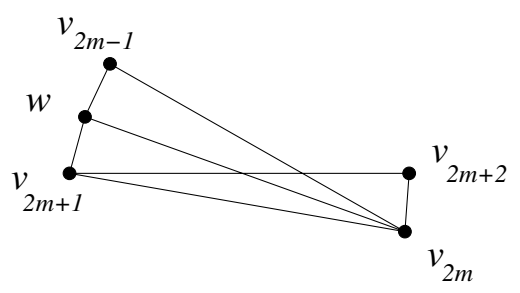

Fig. 6

where

$$
\theta=\frac{\alpha_{m}}{2}=\sin ^{-1}\left(\frac{\left\|v_{2 m-1}-v_{2 m+1}\right\|}{2}\right)
$$

and $\left\|v_{2 m-1}-v_{2 m+1}\right\|$ denotes the distance between $v_{2 m-1}$ and $v_{2 m+1}$. Therefore, the perimeter of the polygon is

$$
L_{\alpha, \beta}=4 \sin \left(\frac{\alpha}{2}\right)+16(m-1) \sin \left(\frac{\beta}{4}\right)+4\left\|v_{2 m+1}-w\right\|+2\left\|v_{2 m+2}-v_{2 m}\right\| .
$$

Choosing $\alpha=\pi / n$ and $\beta=2 \pi / n$ produces a polygon whose perimeter differs from the upper bound $\bar{L}_{n}$ by $O\left(1 / n^{3}\right)$, so this is slightly worse than $T_{n}$. However, we can improve the perimeter by making small adjustments to the parameters. Studying the asymptotic expansions shows that choosing $\alpha=\pi / n+\pi^{2} / 2 n^{2}$ and maintaining $\beta=$ $2 \pi / n$ produces polygons with an error term of just $O\left(1 / n^{5}\right)$, and a further adjustment optimizes the coefficient of this term. Selecting

$$
\alpha=\frac{\pi}{n}+\frac{\pi^{2}}{2 n^{2}}-\frac{\pi^{2}}{n^{3}}
$$

and

$$
\beta=\frac{2 \pi}{n}-\frac{2 \pi^{2}}{n^{3}}
$$

produces a family of polygons $V_{n}$ whose perimeters satisfy

$$
\bar{L}_{n}-L\left(V_{n}\right) \sim \frac{\pi^{5}}{16 n^{5}} .
$$

Last, we verify that the polygon $V_{n}$ has diameter 1 . Let $V_{n}^{\prime}$ denote the convex hull of the $(n / 2)+1$ vertices of $V_{n}$ lying on the circuit in its diameter graph. We verify first that $V_{n}^{\prime}$ has unit diameter by using the same strategy employed to check the polygons $S_{n}$ in the proof of Theorem 1. We then observe that a Reuleaux polygon of unit width may be circumscribed about $V_{n}^{\prime}$ by connecting its vertices with circular arcs. Then $V_{n}$ may be inscribed in this Reuleaux polygon as well, so $V_{n}$ has unit diameter.

The values of $L\left(V_{8}\right), L\left(V_{16}\right)$, and $L\left(V_{32}\right)$ are included in Table 7. We venture that $U_{16}$ is the optimal hexadecagon in the isodiametric problem for the perimeter, and ask if the maximal perimeter when $n=2^{m}$ is always achieved by a polygon with the diameter graph of $V_{n}$. 


\section{Acknowledgments}

I thank the referees for their careful reading of the manuscript and for several helpful suggestions.

\section{References}

1. C. Audet, P. Hansen, and F. Messine, The small octagon with longest perimeter, J. Combin. Theory Ser. A, in press.

2. C. Audet, P. Hansen, F. Messine, and S. Perron, The minimum diameter octagon with unit-length sides: Vincze's wife's octagon is suboptimal, J. Combin. Theory Ser. A 108 (2004), 63-75.

3. C. Audet, P. Hansen, F. Messine, and J. Xiong, The largest small octagon, J. Combin. Theory Ser. A 98 (2002), 46-59.

4. L. Bieberbach, Über eine Extremaleigenschaft des Kreises, Jahresber. Deutsch. Math.-Verein. 24 (1915), 247-250.

5. H. Bieri, Ungelöste Probleme: Zweiter Nachtrag zu Nr. 12, Elem. Math. 16 (1961), 105-106.

6. H. T. Croft, K. J. Falconer, and R. K. Guy, Unsolved Problems in Geometry, Problem Books in Mathematics, Vol. 2, Springer-Verlag, New York, 1991.

7. R. L. Graham, The largest small hexagon, J. Combin. Theory Ser. A 18 (1975), 165-170.

8. H. Lenz, Ungelöste Probleme: Nr. 12, Elem. Math. 11 (1956), 86.

9. H. Lenz, Zerlegung ebener Bereiche in konvexe Zellen von möglichst kleinem Durchmesser, Jahresber. Deutsch. Math.-Verein. 58 (1956), 87-97.

10. M. J. Mossinghoff, A \$1 problem, Amer. Math. Monthly 113 (2006), 385-402.

11. K. Reinhardt, Extremale Polygone gegebene Durchmessers, Jahresber. Deutsch. Math.-Verein. 31 (1922), 251-270.

12. A. Rosenthal and O. Szász, Eine Extremaleigenschaft der Kurven konstanter Breite, Jahresber. Deutsch. Math.-Verein. 25 (1916), 278-282.

13. J. J. Schäffer, Ungelöste Probleme: Nachtrag zu Nr. 12, Elem. Math. 13 (1958), 85-86.

14. N. K. Tamvakis, On the perimeter and the area of the convex polygons of a given diameter, Bull. Soc. Math. Grèce (N.S.) 28 (1987), 115-132.

15. S. Vincze, On a geometrical extremum problem, Acta Sci. Math. Szeged 12 (1950), 136-142.

Received July 22, 2005, and in revised form December 29, 2005. Online publication April 18, 2006. 